\title{
Fc-fusion technology and recombinant FVIII and FIX in the management of the hemophilias
}

This article was published in the following Dove Press journal:

Drug Design, Development and Therapy

28 March 2014

Number of times this article has been viewed

\author{
Maria Elisa Mancuso' \\ Pier Mannuccio Mannucci ${ }^{2}$ \\ 'Angelo Bianchi Bonomi Hemophilia \\ and Thrombosis Center, ${ }^{2}$ Scientific \\ Direction, Fondazione IRCCS \\ Ca' Granda, Ospedale Maggiore \\ Policlinico, Milan, Italy
}

\begin{abstract}
Prophylaxis with regular infusions of factor VIII (FVIII)- or factor IX (FIX)- containing products is the mainstay of modern hemophilia care. However, this therapeutic regimen is inconvenient, requiring repeated intravenous injections from childhood. Approaches meant to prolong the half-life of FVIII and FIX in plasma have been developed in order to improve the feasibility and acceptability of replacement therapy, extending protection from bleeding, reducing infusion frequency and hence the need for venous access devices in young children. Several strategies have been implemented to enhance the pharmacokinetics of clotting factors, including conjugation with polyethylene glycol and the production by genetic engineering of fusion proteins containing the coagulation factors linked to a long-lived plasma protein such as albumin or the Fc fragment of immunoglobulin $(\mathrm{Ig}) \mathrm{G}$. The latter technology is one of the most promising, since the prolongation of FVIII and FIX half-life is obtained by exploiting the physiological binding of the $\mathrm{Fc}$ domain to the neonatal $\mathrm{Fc}$ receptor. Fc fusion monomers have been obtained with both recombinant FVIII (rFVIIIFc) and FIX (rFIXFc), and data from preclinical and clinical studies showed improved pharmacokinetics for both factors, which are produced in human embryonic kidney (HEK) 293 cells, thus ensuring full human post-translational modifications. In Phase I/IIa studies, rFVIIIFc and rFIXFc showed 1.5-1.7 fold and 3.0-4.0 fold longer elimination half-life, respectively. Similar data have been obtained in the Phase III clinical studies with rFVIIIFc and rFIX-Fc published recently. Both drugs were satisfactorily safe, particularly with respect to immunogenicity, and no serious adverse event was observed.
\end{abstract}

Keywords: factor VIII, factor IX, long-acting molecules

\section{Introduction}

Hemophilia A and B are congenital bleeding disorders caused by mutations in the genes coding for coagulation factor VIII (FVIII) and factor IX (FIX). ${ }^{1}$ Severe hemophilia, characterized by the complete plasma deficiency of these coagulation factors (less than $1 \%$ ), is epitomized by limb- or life-threatening clinical manifestations such as hemarthrosis, soft-tissue hematomas, retroperitoneal, intracerebral, and excessive postsurgical hemorrhages. Recurrent joint bleeding and soft-tissue hematomas may cause severe arthropathy, muscle contractures, and pseudotumors, leading to chronic pain and disability that often warrant major orthopedic surgery. ${ }^{1}$ Prophylaxis of bleeds, involving the regular infusion of plasma-derived or recombinant pharmaceutical products containing the deficient coagulation factor, is the mainstay of hemophilia care, made evidence-based by the results of two randomized clinical trials that showed the capacity of this therapeutic approach to avoid arthropathy. ${ }^{2,3}$ However, the practical adoption and implementation of prophylaxis is inconvenient, because the most widely used
Correspondence: Maria Elisa Mancuso Angelo Bianchi Bonomi Hemophilia and Thrombosis Center, Fondazione IRCCS Ca' Granda, Ospedale Maggiore Policlinico, Via Pace 9 - 20I22, Milan, Italy Tel +390255034072

Fax +39025 5032072

Email elisamancuso@tiscali.it (c) (i) (-) 2014 Mancuso and Mannucci. This work is published by Dove Medical Press Limited, and licensed under Creative Commons Attribution - Non Commercial (unported, v3.0) license. The full terms of the License are available at http://creativecommons.org/licenses/by-nc/3.0/. Non-commercial uses of the work are permitted lim a dhe werk is properly attributed. Permissions beyond the scope of the License are administered by Dove Medical Press Limited. Information on how to request permission may be found at: http://www.dovepress.com/permissions.php 
therapeutic regimens involve repeated intravenous infusions of the deficient coagulation factors FVIII or FIX in order to maintain plasma trough levels at or above $1 \% .{ }^{4}$ Prophylaxis is usually delivered according to regimens based upon 2-3 injections per week, or even more frequently, owing to the short half-life (10-14 hours) of FVIII products currently available (slightly longer for FIX products, 15-20 hours). ${ }^{5}$ This need creates problems of venous access, mainly but not only in young children, who often require an alternative access such as central venous lines or arteriovenous fistulae. ${ }^{6}$ In addition, the inconvenience of frequent venipunctures is perhaps one of the reasons why many patients quit continuous prophylaxis at the time of adolescence or adulthood.

With this background, approaches meant to prolong the half-life of coagulation factors in plasma have been developed, in order to improve the feasibility and acceptability of replacement therapy. The potential benefits of the therapeutic enhancement brought about by longer-acting coagulation factors would include extended protection from bleeding and reduced infusion frequency, hence less need for venous access devices. Besides conjugation with polyethylene glycol, genetic fusion to plasma proteins is one of the most promising strategies developed in order to produce long-acting FVIII and FIX. It involves engineering of fusion constructs of these coagulation proteins with either albumin or immunoglobulin (Ig)G. The Fc domain of IgG is engineered to form continuous polypeptides with clotting factors that remain longer in plasma because they are cleared more slowly than the native factor and recycled back into the circulation. So far, this approach has been pursued for both FVIII and FIX, and data from studies in patients with hemophilia are already available, ${ }^{7-10}$ even though at the time of writing no product is yet licensed by regulatory agencies in Europe and in the USA. The purpose of this paper is to review the available knowledge on Fc fusion technology in the treatment of hemophilia A and B.

\section{Fc fusion technology}

The Fc domain of immunoglobulins has been previously employed to form fusions with such molecules as cytokines, growth factors, or other proteins used as research tools or therapeutic agents. Fc fusion is an established technology previously used to prolong the half-life of several drugs licensed for the long-term treatment of a number of chronic diseases. ${ }^{11-13}$ Fusion of the Fc domain of IgG to a therapeutic protein prolongs its half-life through binding to the neonatal Fc receptor (FcRn), which is expressed in the epithelial cells lining the intestine, lung, and kidney, with a degree of expres- sion that is relatively constant throughout life in humans. ${ }^{14-16}$ FcRn is also expressed in the endothelial cells lining the vasculature, the site at which IgG protection most likely occurs. ${ }^{17}$ Fc domain binding to FcRn delays lysosomial degradation and favors recycling back into the circulation. ${ }^{18,19}$ Binding with the FcRn is a pH-dependent process, because it occurs intracellularly in acidic endosomes. IgG or Fc fusion proteins are taken up from circulation by nonspecific endocytosis; when the endosomes fuse back to the cell membrane, the neutral $\mathrm{pH}$ causes the dissociation of the Fc domain from FcRn, thereby releasing the IgG or Fc fusion protein into the bloodstream and thus escaping endosomal/lysosomial degradation. ${ }^{20}$ Ultimately, the Fc fragment degrades and does not accumulate in the body. ${ }^{21}$

The Fc domain is a dimer able to bind two molecules of a given therapeutic protein, and first-generation dimeric Fc fusion molecules were successfully used and demonstrated biological activity. However, because there is an inverse relationship between molecular weight and transport efficacy, which is more evident at molecular weights greater than $30 \mathrm{kDa},{ }^{22}$ continuing efforts to improve transport efficiency led to the development of second-generation Fc fusion molecules that are dimeric with respect to the Fc domain but monomeric with respect to the therapeutic protein. Referred to as Fc fusion monomers, these molecules have improved properties compared with their dimeric counterparts when the effector molecule is naturally a monomer, because the monomeric configuration improves transport efficiency and provides a greater extension of the circulating half-life. Moreover, many Fc fusion monomers showed enhanced biologic activity if compared with the dimeric configuration. ${ }^{23,24}$ In particular, pertaining to the FIX Fc fusion protein, the FIXFc monomer has enhanced pharmacokinetic properties compared with the FIXFc dimer, especially with respect to maximum concentration (Cmax) and area under the curve (AUC), ${ }^{24}$ eventually resulting in a prolonged half-life.

\section{rFVIIIFc and rFIXFc development}

High gene expression, efficient secretion into the cell culture medium, and protein-A affinity purification of Fc fusion proteins render simple their downstream manufacturing processes, by allowing the application of the procedures established for antibody drug manufacturing. ${ }^{25}$ Using this technology, rFVIIIFc and rFIXFc were developed by fusing a single molecule of $\mathrm{B}$ domain-deleted (BDD) recombinant FVIII (rFVIII) or FIX (rFIX) to the dimeric Fc domain of IgG1 with no linker sequence. ${ }^{24,26,27}$ Both drugs are produced by recombinant DNA technology in human embryonic kidney 
(HEK) 293 cells that, at variance with nonhuman mammalian cells, ensure full post-translational modifications, including glycosylation. ${ }^{28}$ The latter is the most critical posttranslational modification of therapeutic proteins because it does influence yield, solubility, stability, clearance rate, and immunogenicity. ${ }^{29}$ In particular, post-translational modifications such as glycosylation, phosphorylation, and tyrosine sulfation are thought to be responsible for the lower in vivo recovery for rFIX in comparison with plasma-derived FIX (pdFIX). ${ }^{30,31}$

\section{Preclinical studies of rFVIIIFc and rFIXFc rFVIIIFc}

The biochemical and functional characterization of rFVIIIFc in comparison with current rFVIII products confirmed that rFVIIIFc contains the expected sequence based on the DNA coding sequence and post-translational modifications, such as N-linked glycosylation and tyrosine sulfation. ${ }^{27} \mathrm{rFVIIIFc}$ has similar in vitro activity to BDD rFVIII, and its specific activity is similar on a molar basis by using a chromogenic assay, proving that the activity of the FVIII moiety is not compromised by $\mathrm{Fc}$ fusion. ${ }^{27}$ Moreover, the in vitro affinity for von Willebrand factor (VWF) was modestly reduced (almost 30\% lower than that of BDD rFVIII), a difference that is probably not relevant for the pharmacokinetic profile of the drug. ${ }^{27} \mathrm{rFVIIIFc}$ has been proven to be as effective as rFVIII in treating tail clip injury in hemophilia A mice and fully corrected the whole blood clotting time in hemophilia A dogs. ${ }^{26}$ Moreover, owing to the twofold prolonged halflife, rFVIIIFc showed extended protection from bleeding as well as prolonged correction of the blood clotting time in hemophilic mice and dogs. ${ }^{26}$ Finally, in FcRn knock-out mice, the half-life of rFVIIIFc was similar to that of rFVIII, confirming the role of FcRn in half-life improvement of the Fc fusion protein. ${ }^{26}$

\section{rFIXFc}

The functional characterization of rFIXFc in several animal models (including normal mice, rats, monkeys, and FIXdeficient mice and dogs) showed a 3.0-4.0 fold longer halflife than unmodified rFIX. ${ }^{24}$ Biochemical characterization by Western blotting confirmed the identity of the FIX protein and peptide mapping showed a gamma-carboxylation pattern similar to that of rFIX, with ten of the 12 sites fully occupied. ${ }^{24}$ Several other post-translational modifications were evaluated and compared with those of rFIX and pdFIX. In general, rFIXFc was comparable with rFIX with respect to Serine (Ser) 158 phosphorylation and Tyrosine (Tyr) 155 sulfation; however, N-linked glycosylation fingerprints indicated that glycans are not fully sialylated. ${ }^{24} \mathrm{O}$-linked glycosylated isoforms of the first epidermal growth factor (EGF) domain were similar to those of rFIX but present at different relative ratios, and the degree of beta-hydroxylation of Aspartate (Asp) 64 was greater than that of rFIX or pdFIX. ${ }^{24}$ The levels of activated FIX were tenfold lower for rFIXFc than for either rFIX or pdFIX. ${ }^{24}$ Finally, in FcRn knockout mice, the half-life of rFIXFc was similar to that of rFIX, confirming the role of FcRn in half-life improvement of the Fc fusion protein. ${ }^{24}$

\section{Pharmacokinetics of rFVIIIFc and rFIXFc rFVIIIFc}

Preclinical data from animal models showed a twofold longer terminal half-life of rFVIIIFc than rFVIII. ${ }^{26}$ The firstin-human Phase I/IIa study (ClinicalTrials.gov identifier: NCT01027377) investigated safety and pharmacokinetic properties of rFVIIIFc in 16 previously treated adult patients with severe hemophilia A (FVIII $<1 \mathrm{IU} / \mathrm{dL}$ ), who received a single dose of rFVIII ( 25 or $65 \mathrm{IU} / \mathrm{kg}$ ), followed by the same dose of rFVIIIFc. ${ }^{7}$ rFVIIIFc showed a $1.5-1.7$ fold longer elimination half-life (18.8 hours for both rFVIIIFc doses versus 12.2 and 11.0 hours for 25 and $65 \mathrm{IU} / \mathrm{kg}$ of rFVIII), a 1.5-1.6 fold lower clearance and a 1.5-1.6 fold higher total systemic exposure. ${ }^{7}$ Dose-dependent peak plasma concentrations and recoveries were similar, and time to reach $1 \%$ FVIII activity was $1.5-1.8$ fold longer with rFVIIIFc than rFVIII across all dosages. ${ }^{7}$ Similar results were subsequently obtained in 28 subjects enrolled in the Phase III clinical study (ClinicalTrials.gov identifier: NCT01181128). rFVIIIFc terminal half-life was significantly longer for rFVIIIFc than for rFVIII (19.0 versus 12.4 hours) and, consistently, time to reach 1\% FVIII activity was 4.9 days as compared with 3.3 days with rFVIII. ${ }^{9}$ As already reported in the Phase I/Ila study, rFVIIIFc half-life was positively correlated with the baseline plasma levels of VWF antigen and the incremental in vivo recovery was comparable to that of rFVIII. ${ }^{7,9}$ Comparative pharmacokinetic data obtained by one-stage FVIII coagulation assay were confirmed by chromogenic assay and rFVIIIFc pharmacokinetic data were highly consistent following repeated doses over 14 weeks of treatment. ${ }^{9}$

\section{rFIXFc}

The half-life of rFIXFc was 3.0-4.0 fold longer than that of rFIX in hemophilic mice and dogs. ${ }^{24}$ The improved 
and prolonged activity was confirmed in the first-inhuman Phase I/IIa study (ClinicalTrials.gov identifier: NCT00716716 $)^{8}$ conducted in 14 previously treated adult patients with severe and moderately severe (FIX $\leq 2 \mathrm{IU} / \mathrm{dL})$ hemophilia B who received a single dose of rFIXFc (12.5, $25,50$, or $100 \mathrm{IU} / \mathrm{kg}) .{ }^{8}$ The terminal half-life was threefold longer than that of rFIX (approximately 19 hours), being on average $53.5,57.6$, and 56.5 hours with 25,50 , and $100 \mathrm{IU} / \mathrm{kg}$, respectively. ${ }^{8}$ The incremental recovery of rFIXFc was 0.93 IU/dL per IU/kg and hence similar to that of pdFIX. ${ }^{8}$ Finally, the average time to reach $1 \%$ trough FIX activity was 7.3 , 10.1 , and 12.3 days after 25,50 , and $100 \mathrm{IU} / \mathrm{kg}$, respectively. ${ }^{8}$ Clearance was reduced to $38 \%$ of the historical value reported with rFIX, and the maximum concentration was reached immediately after injection of rFIXFc, suggesting a rapid onset of action similar to that of rFIX. ${ }^{8}$ Data from the Phase III study (ClinicalTrials.gov identifier: NCT01027364), ${ }^{10}$ recently fully published, confirm the pharmacokinetic profile of rFIXFc on a larger patient sample size $(n=123)$, being the mean half-life of rFIXFc 82.1 versus 33.8 hours for rFIX and the mean time to reach $1 \%$ trough FIX activity (after a $50 \mathrm{IU} / \mathrm{kg}$ dose) was 11.2 days. ${ }^{10}$ The pharmacokinetics of rFIXFc was stable over repeated dosing during several weeks, with a significant increase of AUC and mean residence time and a significant decreased clearance for rFIXFc in comparison with that for $\operatorname{rFIX}(P<0.001) .{ }^{10}$

\section{Clinical development of rFVIIIFc and rFIXFc rFVIIIFc}

The results of an open-label, multicenter Phase III study (A-LONG Study; ClinicalTrials.gov identifier: NCT01181128), ${ }^{9}$ designed to evaluate safety, pharmacokinetics, and efficacy of rFVIIIFc in previously treated patients with severe hemophilia A, has been published..$^{9}$ The study enrolled 165 patients who received rFVIIIFc according to three different treatment regimens: 118 of them received regimens of individualized prophylaxis consisting of $25 \mathrm{IU} / \mathrm{kg}$ on day 1 and $50 \mathrm{IU} / \mathrm{kg}$ on day 4 to start, followed by $25-65 \mathrm{IU} / \mathrm{kg}$ every 3-5 days; 24 received prophylaxis with $65 \mathrm{IU} / \mathrm{kg}$ once weekly; and 23 received 10-50 IU/kg (depending on the bleeding severity) for on-demand treatment of bleeding episodes. ${ }^{9}$ The study was finished upon completion of the scheduled pharmacokinetic assessment and achievement of the prespecified number of rFVIIIFc exposure days (EDs), required in order to ensure an acceptable degree of inhibitor detection (ie, a minimum of 104 cases from any treatment arm with at least 50 EDs). ${ }^{9}$ Overall, 153 patients (93\%) com- pleted the study, and 111 of them had more than 50 EDs. ${ }^{9}$ The median annualized bleeding rate was 1.6, 3.6, and 33.6 in each of the three treatment arms, respectively, with a clear advantage for patients treated on prophylaxis versus those treated on demand. ${ }^{9}$ On average, patients treated with individualized prophylaxis received a median weekly dose of $77.7 \mathrm{IU} / \mathrm{kg}$ at a median dosing interval of 3.5 days; with this regimen, $45 \%$ of patients had no bleeds versus the $17 \%$ bleedfree rate observed in the weekly prophylaxis arm. ${ }^{9}$ Across all arms, 757 bleeding episodes were treated with rFVIIIFc at a median dose of $27 \mathrm{IU} / \mathrm{kg}$. The efficacy rate was $87 \%$ with one injection and $98 \%$ with two injections. ${ }^{9}$ Efficacy was also assessed in the surgical setting, and was found to be excellent in eight of nine major surgical procedures performed in nine patients. ${ }^{9}$ With respect to safety, no neutralizing inhibitors were detected in any subject, but five subjects tested positive for low-titer non-neutralizing antibodies directed against FVIII, but not against Fc. ${ }^{9}$ Adverse events were reported in 108 subjects, but only in ten of them were they related to the investigational drug; arthralgia and malaise were the most common. ${ }^{9}$ No thrombotic events, hypersensitivity reactions, and/or anaphylaxis were reported. ${ }^{9}$

The Phase III pediatric study (Kids A-LONG Study, ClinicalTrials.gov identifier: NCT01458106) ${ }^{32}$ is currently recruiting children with severe hemophilia $\mathrm{A}$ aged less than 12 years already exposed to FVIII for a minimum of 50 EDs. This study will assess the safety, efficacy, and pharmacokinetics of rFVIIIFc in approximately 50 children with severe hemophilia A with a prophylactic treatment regimen. Patients who complete the aforementioned Phase III studies have the opportunity to continue treatment with rFVIIIFc in the frame of the ASPIRE extension study (ClinicalTrials.gov identifier: NCT01454739) ${ }^{33}$ that will assess long-term safety and efficacy of rFVIIIFc.

\section{rFIXFc}

An open-label, multicenter Phase III study (B-LONG Study; ClinicalTrials.gov identifier: NCT01027364), ${ }^{10}$ aimed at evaluating safety, pharmacokinetics, and efficacy of rFIXFc in previously treated patients with severe and moderately severe hemophilia B was recently completed..$^{10}$ The study enrolled 123 patients who received rFIXFc according to three different treatment regimens: 61 of them received weekly prophylaxis starting at $50 \mathrm{IU} / \mathrm{kg}$, with subsequent pharmacokinetic-driven dose adjustments; 29 received prophylaxis at individualized intervals, with $100 \mathrm{IU} / \mathrm{kg}$ starting at every 10 day interval and subsequent adjustment of dosing intervals driven by pharmacokinetic data; and 27 received $20-100 \mathrm{IU} / \mathrm{kg}$ 
(depending on the severity of bleeding) for on-demand treatment of bleeds. ${ }^{10}$ Twelve patients (including eight from the three aforementioned treatment groups) received rFIXFc to cover major surgical procedures. ${ }^{10}$ Overall, 115 patients (93\%) completed the study. ${ }^{10}$ The median annualized bleeding rate was $2.95,1.4$, and 17.7 in each of the three treatment arms, respectively, with a clear advantage for patients treated on prophylaxis versus those treated on demand. ${ }^{10}$ The dosing interval for patients treated with individualized prophylaxis increased from the initial 10 days to an average interval $\geq 14$ days in $54 \%$ of cases. ${ }^{10}$ Across all arms, 636 bleeding episodes were treated in 89 patients with rFIXFc at a median dose of $46 \mathrm{IU} / \mathrm{kg} .{ }^{10}$ Across all regimens, the efficacy rate was $90 \%$ with one injection and $>97 \%$ with two injections. ${ }^{10}$ Efficacy was also assessed in the surgical setting, being excellent in 13 of 14 major surgical procedures performed in 12 patients. ${ }^{10}$ A single injection of rFIXFc at a median dose of $91 \mathrm{IU} / \mathrm{kg}$ was required in $86 \%$ of surgeries to maintain hemostasis during the procedure..$^{10}$ Most procedures required up to two injections on the day of surgery, and the majority of patients required no more than three injections during postoperative days $1-3 .{ }^{10}$ With respect to safety, no inhibitors were detected in any subject, and only one serious adverse event (obstructive uropathy) was assessed as possibly related to treatment. ${ }^{10}$

The Phase III pediatric study (Kids B-LONG Study, ClinicalTrials.gov identifier: NCT01440946) $)^{34}$ is currently recruiting children with severe hemophilia B aged less than 12 years who have already been exposed to FIX for a minimum of 50 EDs. This study will assess the safety, efficacy, and pharmacokinetics of rFIXFc used as prophylactic treatment in approximately 26 children with severe hemophilia B. Patients who completed or will complete the aforementioned Phase III studies have the opportunity to continue treatment with rFIXFc in the frame of the B-YOND extension study (ClinicalTrials.gov identifier: NCT01425723) (35 $^{35}$ that will assess long-term safety and efficacy of rFIXFC.

\section{Conclusion}

The evidence summarized above indicates that in the frame of pharmacokinetic studies coagulation FVIII and FIX produced by Fc fusion technology have a longer plasma half-life than standard factor products, although to a much lower extent for FVIII than for FIX..$^{7-10}$ Consistent with the pharmacokinetic data obtained in Phase I-II studies, published clinical data indicate that it is possible to space factor infusions of at least 3 days in the frame of prophylaxis. Hence, there is some degree of sparing venipunctures compared with standard FVIII, but the difference is not dramatic. On the other hand, pharmacokinetic data for rFIXFc should allow a higher degree of infusion spacing. For instance, in patients with hemophilia A it can be predicted that replacement therapy with FVIII can be given every third day instead of every other day, totaling 121 intravenous injections annually instead of 182 . By the same token, in hemophilia B FIX replacement would be given on average every 10 days instead of every third day, totaling 36 annual infusions instead of 121. The available pharmacokinetic and clinical studies indicate that there were no significant adverse events related to the clinical use of these products. It is not surprising that there were very few adverse reactions of the allergic type, because both the components of the fusion polypeptides are plasma proteins with no foreign chemical agent added. Of course, the main theoretical concern before their use in humans was that these highly engineered protein constructs might be seen as foreign by the immune system and thereby cause the new occurrence of inhibitory antibodies even in previously treated patients; ie, those included in Phase I/IIa and Phase III studies. The currently available data allow to exclude that these products carry a strong immunogenicity signal. ${ }^{7-10}$ On the other hand, these favorable results should be regarded as preliminary and long-term surveillance on inhibitor onset is warranted when these products are licensed, as they may become widely available and more patients may switch to their use from the currently available standard products. It will also be important to collect postmarketing data in previously untreated patients with severe hemophilia; ie, the population at higher risk of inhibitor development.

What other problems require more clinical data to be solved? As mentioned above, Fc fusion technology did not prolong the half-life of FVIII and FIX to the same extent. A possible explanation for that is the tight association of rFVIIIFc with VWF, because unlike FIX, FVIII circulates in complex with VWF which has a relatively short terminal half-life of approximately 18 hours. We suspect that the physiological need for FVIII to form complexes with VWF, is a major limitation for the degree to which the half-life of rFVIII products can be extended. In addition, it is not yet clear whether or not there will be problems in the laboratory assay of the fusion protein both in vitro and ex vivo, perhaps due to its variable interaction with phospholipids in the activated partial thromboplastin time (aPTT) reagents used to measure FVIII in patient plasma.

A big issue will of course be the price of these novel products, which is not yet known at the time of writing. Perhaps simplistically, we would suggest that the price should 
be that of the standard products added with that of actual factor doses that are spared owing to the longer half-life, plus a bonus for the research that led to the development of these truly innovative coagulation factors. It is necessary to develop a model for assessing cost-effectiveness, with quality of life measurement being an essential component of this. It is difficult, lacking head-to-head comparisons, to establish how the Fc fusion factors compare with other longacting products that are in the pipeline. The albumin fusion FIX product seems to be appealing, ${ }^{36}$ because albumin is a physiological component of plasma, like IgG. In principle, these two products should be considered more attractive than those based upon the use of polyethylene glycol, because the latter chemical may theoretically accumulate in the body in lifelong-treated patients, like those with severe hemophilia.

All in all, we believe that Fc-fusion products are an exciting innovation in the field of hemophilia treatment, even though only more widespread field use will answer the many still unresolved questions.

\section{Disclosure}

The authors report no conflicts of interest in this work.

\section{References}

1. Mannucci PM, Tuddenham EG. The hemophilias - from royal genes to gene therapy. N Engl J Med. 2001;344(23):1773-1779.

2. Manco-Johnson MJ, Abshire TC, Shapiro AD, et al. Prophylaxis versus episodic treatment to prevent joint disease in boys with severe hemophilia. N Engl J Med. 2007;357(6):535-544.

3. Gringeri A, Lundin B, von Mackensen S, Mantovani L, Mannucci PM; ESPRIT Study Group. A randomized clinical trial of prophylaxis in children with hemophilia A (the ESPRIT Study). J Thromb Haemost. 2011;9(4):700-710.

4. Collins PW, Blanchette VS, Fischer K, et al; rAHF-PFM Study Group. Break-through bleeding in relation to predicted factor VIII levels in patients receiving prophylactic treatment for severe hemophilia A. J Thromb Haemost. 2009;7(3):413-420.

5. Collins PW, Björkman S, Fischer K, et al. Factor VIII requirement to maintain a target plasma level in the prophylactic treatment of severe hemophilia A: influences of variance in pharmacokinetics and treatment regimens. J Thromb Haemost. 2010;8(2):269-275.

6. Valentino LA, Kawji M, Grygotis M. Venous access in the management of hemophilia. Blood Rev. 2011;25(1):11-15.

7. Powell JS, Josephson NC, Quon D, et al. Safety and prolonged activity of recombinant factor VIII Fc fusion protein in hemophilia A patients. Blood. 2012;119(13):3031-3037.

8. Shapiro AD, Ragni MV, Valentino LA, et al. Recombinant factor IX-Fc fusion protein (rFIXFc) demonstrates safety and prolonged activity in a phase $1 / 2$ a study in hemophilia B patients. Blood. 2012;119(3):666-672.

9. Mahlangu J, Powell JS, Ragni MV, et al. Phase 3 study of recombinant factor VIII Fc fusion protein in severe hemophilia A. Blood. 2014;123:317-325

10. Powell JS, Pasi KJ, Ragni MV, et al. Phase 3 study of recombinant factor IX Fc fusion protein in hemophilia B. $N$ Engl J Med. 2013;369:2313-2323.
11. Fisher CJ, Agosti JM, Opal SM, et al. Treatment of septic shock with the tumor necrosis factor receptor:Fc fusion protein. The Soluble TNF Receptor Sepsis Study Group. N Engl J Med. 1996;334(26):1697-1702.

12. Ellis CN, Krueger GG; Alefacept Clinical Study Group. Treatment of chronic plaque psoriasis by selective targeting of memory effector T lymphocytes. N Engl J Med. 2001;345(4):248-255.

13. Genovese MC, Becker JC, Schiff M, et al. Abatacept for rheumatoid arthritis refractory to tumor necrosis factor alpha inhibition. $N$ Engl J Med. 2005;353(11):1114-1123.

14. Israel EJ, Taylor S, Wu Z, et al. Expression of the neonatal Fc receptor, FcRn, on human intestinal epithelial cells. Immunology. 1997;92(1): 69-74.

15. Spiekermann GM, Finn PW, Ward ES, et al. Receptor-mediated immunoglobulin $\mathrm{G}$ transport across mucosal barriers in adult life: functional expression of FcRn in the mammalian lung. J Exp Med. 2002;196(3): 303-310.

16. Haymann JP, Levraud JP, Bouet S, et al. Characterization and localization of the neonatal Fc receptor in adult human kidney. J Am Soc Nephrol. 2000;11(4):632-639.

17. Antohe F, Rădulescu L, Gafencu A, Gheție V, Simionescu M. Expression of functionally active FcRn and the differentiated bidirectional transport of IgG in human placental endothelial cells. Hum Immunol. 2001;62(2):93-105.

18. Ward ES, Zhou J, Ghetie V, Ober RJ. Evidence to support the cellular mechanism involved in serum IgG homeostasis in humans. Int Immunol. 2003;15(2):187-195.

19. Ober RJ, Martinez C, Vaccaro C, Zhou J, Ward ES. Visualizing the site and dynamics of IgG salvage by the MHC class I-related receptor, FcRn. J Immunol. 2004;172(4):2021-2029.

20. Lencer WI, Blumberg RS. A passionate kiss, then run: exocytosis and recycling of IgG by FcRn. Trends Cell Biol. 2005;15(1):5-9.

21. McGarry T, Hough R, Rogers S, Rechsteiner M. Intracellular distribution and degradation of immunoglobulin $\mathrm{G}$ and immunoglobulin G fragments injected into HeLa cells. J Cell Biol. 1983;96(2): 338-346.

22. Desai MA, Mutlu M, Vadgama P. A study of macromolecular diffusion through native porcine mucus. Experientia. 1992;48(1):22-26.

23. Bitonti AJ, Dumont JA, Low SC, et al. Pulmonary delivery of an erythropoietin $\mathrm{Fc}$ fusion protein in non-human primates through an immunoglobulin transport pathway. Proc Natl Acad Sci U SA. 2004;101(26): 9763-9768.

24. Peters RT, Low SC, Kamphaus GD, et al. Prolonged activity of factor IX as a monomeric Fc fusion protein. Blood. 2010;115(10):2057-2064.

25. Goldenberg MM. Etanercept, a novel drug for the treatment of patients with severe, active rheumatoid arthritis. Clin Ther. 1999;21(1):75-87; discussion 1-2.

26. Dumont JA, Liu T, Low SC, et al. Prolonged activity of a recombinant factor VIII-Fc fusion protein in hemophilia A mice and dogs. Blood. 2012;119(13):3024-3030.

27. Peters RT, Toby G, Lu Q, et al. Biochemical and functional characterization of a recombinant monomeric factor VIII-Fc fusion protein. J Thromb Haemost. 2013;11(1):132-141.

28. Swiech K, Picanço-Castro V, Covas DT. Human cells: new platform for recombinant therapeutic protein production. Protein Expr Purif. 2012;84(1):147-153.

29. Ghaderi D, Zhang M, Hurtado-Ziola N, Varki A. Production platforms for biotherapeutic glycoproteins. Occurrence, impact, and challenges of non-human sialylation. Biotechnol Genet Eng Rev. 2012;28: $147-175$.

30. White GC 2nd, Beebe A, Nielsen B. Recombinant factor IX. Thromb Haemost. 1997;78(1):261-265.

31. Roth DA, Kessler CM, Pasi KJ, Rup B, Courter SG, Tubridy KL; Recombinant Factor IX Study Group. Human recombinant factor IX: safety and efficacy studies in hemophilia B patients previously treated with plasma-derived factor IX concentrates. Blood. 2001;98(13): 3600-3606. 
32. Biogen Idec. Study of Recombinant Coagulation Factor VIII Fc Fusion Protein, BIIB 031, in Pediatric PTP Subjects With Hemophilia A. Updated December 12, 2013. Available from: http://clinicaltrials. gov/show/NCT01458106. NLM identifier: NCT01458106. Accessed February 17, 2014.

33. ClinicalTrials.gov. Long-Term Safety and Efficacy of Recombinant Human Coagulation Factor VIII Fusion Protein (rFVIIIFc) in the Prevention and Treatment of Bleeding Episodes in Previously Treated Subjects With Hemophilia A. Last updated September 12, 2013. Available from: http://clinicaltrials.gov/show/NCT01454739.

34. ClinicalTrials.gov. Study of Recombinant Coagulation Factor IX Fc Fusion Protein, BIIB 029, in Pediatric PTP Subjects With Hemophilia B. Updated September 12, 2013. Available from: http://clinicaltrials. gov/show/NCT01440946.
35. ClinicalTrials.gov. Long-Term Safety and Efficacy of Recombinant Human Coagulation Factor IX Fusion Protein $(\mathrm{rFIXFc})$ in the Prevention and Treatment of Bleeding Episodes in Previously Treated Subjects With Hemophilia B (B-YOND). Last updated September 12, 2013. Available from: http://clinicaltrials.gov/show/NCT01425723.

36. Santagostino E, Negrier C, Klamroth R, et al. Safety and pharmacokinetics of a novel recombinant fusion protein linking coagulation factor IX with albumin (rIX-FP) in hemophilia B patients. Blood. 2012;120(12):2405-2411.

\section{Publish your work in this journal}

Drug Design, Development and Therapy is an international, peerreviewed open-access journal that spans the spectrum of drug design and development through to clinical applications. Clinical outcomes, patient safety, and programs for the development and effective, safe, and sustained use of medicines are a feature of the journal, which has also been accepted for indexing on PubMed Central. The manuscript management system is completely online and includes a very quick and fair peer-review system, which is all easy to use. Visit http://www.dovepress.com/testimonials.php to read real quotes from published authors.

Submit your manuscript here: http://www.dovepress.com/drug-design-development-and-therapy-journal 